\title{
New theories and methods for technology adoption research
}

\author{
Robert J. Kauffman • \\ Angsana A. Techatassanasoontorn
}

Published online: 5 November 2011

(C) Springer Science+Business Media, LLC 2011

This special issue includes six articles on different aspects of technology adoption that represent the development and application of different theoretical and methodological approaches to the business problems that they treat. In terms of theory, three of the articles use behavioral and organizational theories, including adaptive structuration theory, management fashion theory, the unified theory of technology acceptance, the technology acceptance model, and diffusion of innovation theory. The other two are based on economic theory, including network effects theory, and economic growth theory. The methods used are also dramatically different in each of the studies. Three studies use field research and survey methods that are common in organizational and behavioral research designs. The authors of these studies are: Sinclaire and Vogus; Park, Lee and Yi; and Hester. Techatassanasoontorn and Suo's study, in contrast, uses agent-based computational simulation and network modeling to produce its results. Westland's article discusses affective information technologies for data acquisition in survey research, and employs a detailed historical overview of the literature, along with survey methods and data analysis to illustrate its application. Finally, the study by Ho, Kauffman and Liang uses panel data econometrics and a variety of refinements that permit the triangulation of evidence based on the assumption of somewhat different modeling relationships. Taken together, the contents reflect some of the current advances that

\section{R. J. Kauffman ( $\square)$}

School of Information Systems, Singapore Management University, 80 Stamford Road, Singapore 198702, Singapore e-mail: rob7585@gmail.com

\section{A. A. Techatassanasoontorn}

Faculty of Business and Law, Auckland University of Technology, Auckland 1142, New Zealand are occurring in the literature with respect to theory and methods for technology adoption research that we hope to showcase in this special issue.

The special issue opens with a piece by Jollean Sinclaire and Clinton E. Vogus on "Adoption of Social Networking Sites: An Exploratory Adaptive Structuration Perspective for Global Organizations." Since social media have become popular with Internet users around the world, global businesses have also recognized their potential importance as a means to be in touch with their customers and the marketplace overall. The theoretical focus of this research involves the dual use of management fashion theory and adaptive structuration theory in order to show the variety of beneficial uses to which organizations are applying social media. Their research is survey-based, and includes interviews with middle to senior managers in a total of 72 large firms that have global operations. In addition to reporting the empirical regularities of social media use, the authors also characterize the different purposes that the organizations have for investing in social media. For example, they report passive versus active applications, proactive versus reactive implementations, and tactical versus strategic uses. The authors also suggest that adaptive structuration theory enables them to interpret the use of social media in global organizations in terms of the low levels of restrictiveness and sophistication, crossed with the open nature of the social media environment on the Internet, to draw conclusions about the basis for further technological innovations with social media, as well as related changes in the way that organizations operate and structure themselves in relation to these technological innovations. Although their study is exploratory and early in its cycle of development, it nevertheless offers a useful perspective on the various issues that can be pursued in follow-on research to gain a fuller understanding of the 
adoption and related outcomes associated with social media and social networking use in global organizations.

The next article is by Sung-Hee Park, Lorraine Lee and Mun Y. Yi, entitled "Group-Level Effects of Facilitating Conditions on Individual Acceptance of Information Systems" The authors seek to expand our understanding of individual level models of technology acceptance, especially the unified theory of acceptance and use of technology model, by supplementing its explanatory capabilities with a group-level variable. That variable is facilitating conditions in the organization, which can be captured by the degree to which an individual believes that an organizational or technical infrastructure exists to support the adoption and use of the system that is under study. So facilitating conditions will involve user perceptions that the organization is putting an appropriate amount of resources to facilitate user adoption and acceptance of a system, such as training or funding, which are critical. This study also integrates the multi-level perspective to explicitly recognize that individual-level and organizational-level variables are of simultaneous importance in explaining adoption behavior. The authors refer to these as conjoint effects. To understand the conjoint effects as well as other factors associated with user adoption in an organizational context, the authors carried out two field surveys involving the rollout of a Web-based comprehensive course management system, and obtained the following findings: individual facilitating and organizational facilitating conditions have separable effects on system adoption and use; and the organizational-level variable explains a greater amount of the variance for individual acceptance than do the individual-level variables.

In "A Comparative Analysis of the Usage and Infusion of Wiki and Non-Wiki-Based Knowledge Management Systems," Andrea Hester focuses on the evaluation of reciprocity expectation and personal innovativeness with information technology as a basis for explaining user acceptance of wiki technology-based knowledge management systems. She inquires about whether the adoption of wiki and non-wiki-based systems involve the same considerations by those who adopt them. She also asks if the dynamics of adoption and subsequent usage are similar across these different kinds of knowledge support mechanisms. She discusses two constructs that have been used in prior research by other authors to extend the theory of technology acceptance. One of her key constructs, reciprocity expectation, is the idea that a person hopes, upon providing his own knowledge to a knowledge management system, that others will reciprocate and share their knowledge so that everyone is better off. The other key construct, personal innovativeness in information technology, previously proposed by Agarwal and Prasad in a 1998 article in Information Systems Research, is the propensity of a person to express a willingness to adopt and use innovative technologies. It implies that the person will have a similar attitude toward wiki-based knowledge management systems, in spite of their relative newness. Hester's survey, based on the responses of 170 subjects, showed that adoption and usage of wiki-based and nonwiki-based knowledge management systems are driven by different factors, and that reciprocity expectations and personal innovativeness with information technology are more relevant for understanding the dynamics of wikibased knowledge management systems than the prior generation of non-wiki-based systems.

Agent-based computational methods related to smallworld and scale-free networks to enhance our knowledge of various technology adoption phenomena are of significant interest among theorists and empiricists in this area of research. The fourth article in the special issue, by Angsana A. Techatassanasoontorn and Shuguang Suo, offers an innovative assessment on product incompatibility and standards in technology industries on some of the longstanding predictions of network economics. Their article is entitled "Influences on Standards Adoption in De Facto Standardization." Its main premise is that the process of de facto standardization that we often see with incompatible technology products, as they build up their installed bases, may not lead to winner-take-all, dominant network outcomes under some circumstances. They assert a new theoretical perspective: a variety of kinds of network-based connections among users may create another source of influence that has the potential to balance out network effects and switching costs, leading to different observed decision-making outcomes in the marketplace. This is an interesting observation because the reigning viewpoint in network economics is that winner-take-all outcomes are the most likely. The authors model the influence of network effects and vendor strategies on switching costs, pricing, and the enhancement of product functionality in two kinds of networks. Their agent-based simulation approach shows that, for small-world and scale-free networks, de facto standardization process doesn't always have a prototypical S-shaped evolutionary pattern-something that marketing and product managers will surely want to keep in mind.

The next to last contribution to the special issue is by J. Christopher Westland, and is entitled "Affective Data Acquisition Technologies in Survey Research." Affective data acquisition technologies involve the use of biometric sensors and other techniques to gauge human behavioral reactions involving feelings and emotions that occur in a variety of social settings. The author provides an historical perspective on the growth, development and adoption of affective computing approaches. He reminds us that this is an important emerging area of technology that should be of interest to IS researchers. He characterizes affective data 
acquisition technologies as being costly, cumbersome and invasive for the people who used it before, with the result that its adoption and diffusion have been slow. The author notes, however, that this situation is changing, as the technologies become more economical and their capabilities become embedded in social networks. The main study focuses on demonstrating the use of an affective technology-based research design for the study of human subjects' reactions to emotionally-charged issues in United States' politics. His results provide a basis for understanding the kinds of issues that people react the most strongly to in affective computing terms, especially economic issues and international conflicts involving wars. He concludes with a discussion of the risks and issues associated with affective computing-based research designs.

The final article of this special issue discusses "InternetBased Selling Technology and E-Commerce Growth: A Hybrid Growth Theory Approach with Cross-Model Inference," co-authored by Shu-Chun Ho, Robert J. Kauffman and Ting-Peng Liang. The authors examine the growth of business-to-consumer (B2C) e-commerce across 42 countries around the world, and evaluate the extent to which the adoption of Internet-based selling technology infrastructure adoption supports the observed growth. To do this, the authors propose a hybrid growththeoretic perspective, which emphasizes the dual roles of exogenous and endogenous factors that influence e-commerce growth. The key exogenous factor is the international openness of a country to external influences, while the endogenous factors include Internet user penetration, capital invested in telecommunications and Internet-based selling technology infrastructure. Their approach to the development of evidence in this research relies upon the estimation of econometric models that represent a variety of error term information structures, as well as the possibility of simultaneity between Internet-based selling technology infrastructure adoption and e-commerce growth. They report three main findings: (1) the proposed hybrid growth model is an effective tool for the estimation of the GDP-normalized level of B2C e-commerce revenues over time; (2) an embedded technology adoption function is a useful modeling addition for the explanation of e-commerce revenue growth; and (3) the availability of venture capital in a country is an effective precursor to the development of relevant technology infrastructure in support of e-commerce activities.

We acknowledge the efforts that the authors made, as we took them through multiple rounds of reviews to develop their papers. The contributions of the reviewers were especially notable, since they offered insights and suggestions that led to the finished research products that you see in this special issue. We also appreciated additional assistance from various people who helped us to ensure there were no conflicts of interests in the work-up of papers involving the guest editors. Finally, we obtained support from Information Technology and Management's editorial office, especially the Journal Manager, Deepika Jothinathan, and its Co-Editor in Chief, Varghese Jacob, and would like to offer thanks for that. 